\title{
Ciclo reproductivo del ostión de manglar Crassostrea rhizophorae (Bivalvia: Ostreidae) en la Bahía de Camamu, Bahia, Brasil
}

\author{
Tiago Lenz \& Guisla Boehs \\ Programa de Postgrado en Sistemas Acuáticos Tropicales-Departamento de Ciências Biológicas/Universidade \\ Estadual de Santa Cruz-Rodovia Ilhéus-Itabuna, km 16, Salobrinho, 45.662-900, Ilhéus, Bahia, Brasil; \\ tiagopesca@hotmail.com,gboehs@uesc.br
}

Recibido 15-X-2009. Corregido 25-VII-2010. Aceptado 31-VIII-2010.

\begin{abstract}
Reproductive cycle of the mangrove oyster Crassostrea rhizophorae (Bivalvia: Ostreidae) in Camamu Bay, Bahia, Brasil. The mangrove oyster Crassostrea rhizophorae is important fishery resource along the entire Brasilian coast with excellent potential for marine culture. The purpose of this paper was to examine the reproductive characteristics of the oyster of the Maraú river estuary in Camamu Bay, Bahia, Brasil. The samples were collected monthly, from September 2006 to August 2007, at two points (I and II) in Camamu Bay. At each site 20 oysters were collected for histological analysis, fixed in Davidson's solution, embedded in paraffin, dehydrated in an ethanol series, sectioned ( $7 \mu \mathrm{m}$ thick) and stained with Harris hematoxylin and Eosin (HE). Additionally, 30 oysters were sampled, at each point, for a condition index analysis. The water temperature ranged from $23.5^{\circ} \mathrm{C}$ to $30^{\circ} \mathrm{C}$ and the salinity from 15 to 25 ups at Point I (Maraú) and from 25 to 35 at Point II (Tanque Island). The oyster's height ranged from 30 to $92 \mathrm{~mm}$ at Point I and from 27 to $102 \mathrm{~mm}$ at Point II, with an average of $49.0 \mathrm{~mm} \pm 9.1(\mathrm{n}=230)$ and $49.9 \mathrm{~mm} \pm 9.9(\mathrm{n}=237)$, respectively. Among the sampled oysters at Point I, 59.1\% were females, $31.3 \%$ males, $1.3 \%$ hermaphrodites and $8.2 \%$ of the oysters of undetermined sex. At Point II, $66.2 \%$ were females, $30.4 \%$ males, $0.8 \%$ hermaphrodites and $2.5 \%(n=237)$ of undetermined sex. The gonadic stage analysis indicated that the reproduction period of the C. rhizophorae in the Maraú Peninsula was continuous all year, without any regressive phase. The condition index (R) ranged from $8.0 \%$ to $17.7 \%$. The peak periods of $\mathrm{R}$ coincided with the expressive oyster's percentage in the maturation and liberation gametic stages. The results of these findings will contribute information for the oyster spat collection and to the process installation of the oyster culture in Camamu Bay. Rev. Biol. Trop. 59 (1): 137-149. Epub 2011 March 01.
\end{abstract}

Key words: Crassostrea, mangrove oysters, reproduction, condition index, oyster culture, Brasil.

El ostión de manglar Crassostrea rhizophorae (Guilding, 1828) es un importante recurso pesquero que se distribuye por toda la costa brasileña, habita en regiones de manglar en ensenadas, bahías y esteros, adheridos a rocas y otros sustratos consolidados (Rios 1994). En los manglares, se encuentra generalmente fijo a las raíces del mangle rojo, Rhizophora mangle, en la zona entre mareas (Bacon 1971, Nascimento 1983).

El ostión de manglar es una de las especies nativas con mayor potencial para la maricultura en la costa brasileña (Pereira et al. 2003, Nascimento \& Pereira 2004).

En Brasil, la ostricultura se basa en $C$. rhizophorae y en $C$. gigas (Thunberg, 1795) que corresponde al $23 \%$ del total de moluscos producidos. La producción nacional de moluscos es pequeña, aproximadamente de 16 mil toneladas por año, con el Estado de Santa Catarina que obtiene $90 \%$ de lo producido (EPAGRI 2007).

Los ostiones del género Crassostrea son hermafroditas secuenciales, siendo la gónada 
primaria bisexual (Galtsoff 1964). En general, empiezan su vida como macho, luego pueden producir oocitos y nuevamente pueden volver a producir espermatozoides (Galtsoff 1964, Andrews 1979). La mayoría de los ejemplares jóvenes alcanzan la madurez sexual antes de alcanzar los 30mm de altura (Vélez 1976, Nascimento et al. 1980).

Se ha caracterizado el ciclo reproductivo y la influencia de las variables abióticas sobre la reproducción de $C$. rhizophorae, en diversas localidades (Vélez 1977, Nascimento \& Lunetta 1978, Galvão et al. 2000). El estudio más detallado sobre el ciclo gonádico de ese ostión en Brasil fue realizado por Nascimento (1978), quién caracterizó los estadios de madurez y las influencias abióticas sobre el proceso reproductivo.

Conocer las características sexuales y los estímulos ambientales que rigen la madurez gonádica y la liberación de gametos es importante para sostener la explotación comercial de moluscos (Cledón et al. 2004), ya que evidencian las épocas de desove y reclutamiento de larvas en las áreas de estudio (Wakamatsu 1973, Galvão et al. 2000).

El rendimiento de la carne, es un índice eco-fisiológico utilizado para evaluar la calidad de la carne y la productividad en cultivos de moluscos bivalvos (Rebelo et al. 2005). Es un importante parámetro para la comercialización y aceptación del producto, ya que puede evidenciar una acumulación de reservas nutritivas para la reproducción (Ruiz et al. 1969, Nascimento \& Pereira 1980a), la presencia de parásitos en los tejidos (Nascimento \& Pereira 1980b) y el efecto de las variaciones de las condiciones ambientales (Wakamatsu 1973, Prieto et al. 1999, Christo 2006).

A través de este estudio, se evaluó la reproducción (proporción sexual y estadios de madurez gonádica) y el rendimiento de la carne del ostión, C. rhizophorae, en el estero del Rio Maraú, Bahía de Camamu (Bahia, Brasil), con el objetivo de iniciar cultivos en la zona mediante el manejo sostenible del recurso en la región.

\section{MATERIALES Y MÉTODOS}

El Complejo delta lagunar de la Bahía de Camamu está ubicado en el Estado de Bahia entre los $13^{\circ} 40^{\prime}-14^{\circ} 12^{\prime} \mathrm{S}-38^{\circ} 55^{\prime}-39^{\circ} 9^{\prime} \mathrm{W}$ y comprende una área total de $15.000 \mathrm{~km}^{2}$, se extiende aproximadamente $130 \mathrm{~km}$ a lo largo del margen continental de la costa este brasileña (Souza-Lima et al. 2003). El sistema hidrográfico que compone la zona de influencia directa de este estudio está constituido por diversas cuencas que alimentan la Bahía de Camamu. El área delta lagunar de ese accidente geográfico está formada por la confluencia de los ríos Maraú, Conduru, Acaraí, Pinaré, Igrapiúna y Serinhaém con el Océano Atlántico (S.E.I. 2000).

El estudio fue realizado en el estero del Río Maraú, en la porción occidental del complejo de la Bahía de Camamu, en los siguientes puntos de muestreo: Punto I, Maraú (1406' $55^{\prime}$ " S - 39 $\left.02^{\prime} 83^{\prime \prime} \mathrm{W}\right)$, ubicado en el sector interno y Punto II, Isla del Tanque (1359'92” S $\left.38^{\circ} 58^{\prime} 17^{\prime \prime} \mathrm{W}\right)$, cerca a la entrada de la bahía. Las recolectas fueron realizadas mensualmente, entre septiembre de 2006 y agosto de 2007. En el momento de la recolección fueron monitoreadas: la temperatura (multiparámetros Horiba) y la salinidad, con un refractómetro óptico manual Atago S/Mill. Los índices pluviométricos para la región fueron obtenidos en la estación de la Comisión Ejecutiva del Plan de la Labora del Cacao (CEPLAC), ubicada en el municipio de Camamu (BA). A través, del Análisis de Variancia (ANOVA, $\alpha=0.05$ ), se buscó detectar variaciones significativas espaciales y temporales en los valores de temperatura y salinidad.

Los animales fueron recolectados al azar de las raíces del manglar rojo $R$. mangle y se utilizaron aquellos ostiones en talla reproductiva, esto es, con altura (eje dorso-ventral) mayor de $30 \mathrm{~mm}$, tamaño en el que la mayoría de los individuos alcanzan la madurez sexual (Vélez 1976, Nascimento et al. 1980).

Para el análisis histológico de las gónadas, se recolectaron muestras mensuales de 15 a 20 animales en cada punto y 467 ejemplares en 
total. También, se obtuvieron mensualmente muestras con cerca de 60 animales en los mismos puntos para el cálculo del rendimiento de la carne (R), totalizando 669 animales. Todos los animales fueron medidos con relación a su altura (eje dorso-ventral), utilizando un Vernier digital, con precisión de $0.01 \mathrm{~mm}$. Las muestras de tejido de cada animal fueron fijadas en solución de Davidson (Shaw \& Battle 1957) por 30 horas y después transferidas a etanol al $70 \%$. Los tejidos fijados fueron preparados de acuerdo con técnicas de histología clásica de inclusión en parafina, secciones tisulares de aproximadamente $7 \mu \mathrm{m}$ de grosor y tinción con hematoxilina de Harris y eosina. Las observaciones fueron efectuadas con un microscopio óptico Olympus en aumentos 4, 10, 40 y 100 X.

Los animales fueron clasificados en: machos, hembras, hermafroditas e indeterminados. Para conocer si la proporción de sexos mensual en cada estación de muestreo y a lo largo del período del trabajo, difiere de la proporción esperada de 1: 1 se utilizó una prueba de $\chi^{2}$ (qui-cuadrado). Para la clasificación de los estadios gonádicos fueron utilizados como referencia los trabajos de Nascimento (1978) y Nascimento \& Lunetta (1978). Como todos los animales utilizados ya habían alcanzado la talla reproductiva, la clasificación utilizada fue en: gametogénesis, madurez, desove parcial de gametos, desove avanzado de gametos, desove con recuperación de los gametos y reposo.

Para la obtención de los datos biométricos del ostión destinados a la evaluación del rendimiento de la carne (R), después de la medición de los ejemplares, se obtuvo el peso individual total y de las partes blandas (carne), con el auxilio de una balanza electrónica de precisión de $0.001 \mathrm{~g}$. El rendimiento de la carne (R) fue calculado a través de la fórmula: $\mathrm{R}(\%)=$ Peso húmedo de las partes blandas/Peso total del animal x 100 .

\section{RESULTADOS}

Parámetros físicos y químicos: La temperatura del agua varió de 24.4 a $30^{\circ} \mathrm{C}$ (promedio=27.5 \pm 2.1 ) en el Punto I y de 23.5 a $30^{\circ} \mathrm{C}$ (promedio $\left.=27.3 \pm 2.2\right)$ en el Punto II $(\mathrm{N} /$ local=12) (Fig. 1a). No hubo variación espaciotemporal significativa de ese factor (ANOVA, p> 0.05). La salinidad varió entre 15 y 25 (promedio $=18.9 \pm 2.9$ ) en el Punto I y de 25 a 35 (promedio $=31.1 \pm 3.2)$ en el Punto II $(\mathrm{N} /$ local=12) con variación significativa (ANOVA, $\mathrm{p}<0.05$ ) entre los sitios (Fig. 1b).

Con relación a la variación pluviométrica, fueron caracterizados como períodos lluviosos (>200mm) octubre y noviembre de 2006 (250.9 y $211.7 \mathrm{~mm}$, respectivamente) y febrero de 2007 (509.6mm), mes en el cual ocurrió la mayor intensidad pluviométrica.

Dimensiones y Proporción Sexual: La altura de los ostiones varió de 30 a $92 \mathrm{~mm}$ en el Punto I y de 27 a $102 \mathrm{~mm}$ en el Punto II, con un promedio de $49 \mathrm{~mm} \pm 9.1 \quad(\mathrm{n}=230)$ y $49.9 \mathrm{~mm} \pm 9.9 \quad(\mathrm{n}=237)$, respectivamente. Las diferencias de altura entre los puntos, no fueron significativas (Prueba t-student, $\mathrm{p}>0.05$ ).

La razón sexual mostró la predominancia de hembras sobre los machos en prácticamente todo el período. En el Punto I se obtuvo la proporción macho: hembra (M:H), de 1:1.9 y en el Punto II de 1:2.1, con semejanza (Prueba qui-cuadrado, $\mathrm{p}>0.05$ ) entre sitios. Sin embargo, en julio de 2007 en el Punto I, se observó una relación inversa (1:0.8) y en enero de 2007 en el Punto II, la proporción fue igual (1:1). En escala temporal, diferencias significativas fueron encontradas en octubre de 2006 y mayo de 2007 en el Punto I (Cuadro 1), y en mayo y agosto de 2007 en el Punto II (Cuadro 2).

La ocurrencia de hermafroditismo fue de $1.3 \%$ en el Punto I y de $0.8 \%$ en el Punto II. Los hermafroditas en ambos puntos fueron encontrados durante noviembre 2006 y febrero 2007.

Fotomicrografías de gónadas de macho, hembra, hermafrodita y de sexo indeterminado componen la Fig. 2.

Ciclo reproductivo: En el Punto I-Maraú, la mayor parte de los animales en gametogénesis fue observada en septiembre de 2006 y julio 2007. Los eventos de desove, con mayor 

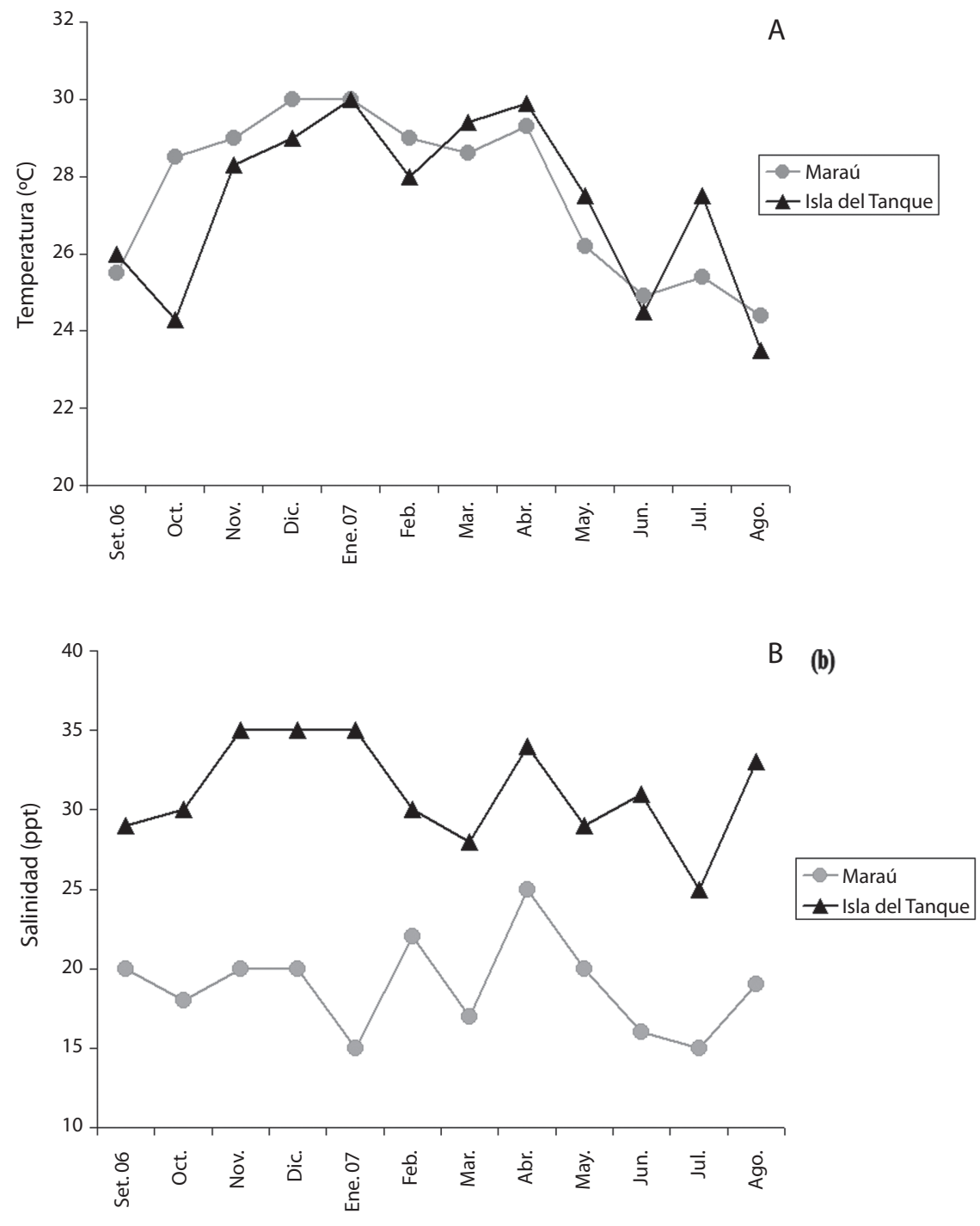

Fig. 1. (a) Valores de temperatura $\left({ }^{\circ} \mathrm{C}\right)$ y (b) salinidad (ups) en el Punto I (Maraú) y en el Punto II (Isla del Tanque), en el estero del Río Maraú, Bahía de Camamu, Bahia, Brasil, septiembre 2006-agosto 2007 (N=12/local).

Fig. 1. (a) Values of the temperature $\left({ }^{\circ} \mathrm{C}\right)$ and (b) salinity (ups) at Point I (Maraú) and Point II (Tanque Island), in the Maraú River estuary, Camamu Bay, Bahia, Brasil, from September 2006 to August 2007 (N=12/point).

presencia de animales maduros, acontecieron en octubre 2006, enero, febrero y agosto 2007. El desove parcial de gametos ocurrió durante todos los meses. El mayor desove con recuperación de gametos se dio en noviembre, diciembre de 2006 y marzo, abril y julio 2007. En el Punto
I-Isla del Tanque, se verificó la mayor parte de los animales en gametogénesis de abril a julio de 2007. Los eventos de desove, con mayor presencia de animales maduros, ocurrieron en septiembre-octubre 2006 y en enero-febrero y agosto 2007. El desove parcial de gametos 
CUADRO 1

Frecuencia absoluta y relativa de machos $(M)$, hembras $(H)$, hermafroditas $(E)$ y de sexo indeterminado $(I)$ del ostión Crassostrea rhizophorae en el Punto I-Maraú, de septiembre de 2006 a agosto 2007

TABLE 1

Absolute and relative frequency of the male $(M)$, female $(H)$, hermaphrodite $(E)$ and indeterminate sex $(I)$ of the Crassostrea rhizophorae oyster in Point I-Maraú, during from September 2006 to August de 2007

$\begin{array}{lccccccccccc}\text { Mes/Año } & \mathrm{M} & \mathrm{H} & \mathrm{M}+\mathrm{H} & \chi^{2} & \mathrm{E} & \mathrm{I} & \text { Total } & \% \mathrm{M} & \% \mathrm{H} & \% \mathrm{E} & \% \mathrm{I} \\ \text { sep/06 } & 5 & 9 & 14 & 1.14 & 0 & 6 & 20 & 25 & 45 & 0 & 30 \\ \text { oct/06 } & 5 & 14 & 19 & 4.26^{*} & 0 & 0 & 19 & 26.31 & 73.68 & 0 & 0 \\ \text { nov/06 } & 5 & 13 & 18 & 3.56 & 1 & 1 & 20 & 25 & 65 & 5 & 5 \\ \text { dec/06 } & 8 & 10 & 18 & 0.22 & 0 & 2 & 20 & 40 & 50 & 0 & 10 \\ \text { ene/07 } & 6 & 10 & 16 & 1 & 0 & 0 & 16 & 37.5 & 62.5 & 0 & 0 \\ \text { feb/07 } & 6 & 11 & 17 & 1.47 & 2 & 1 & 20 & 30 & 55 & 10 & 5 \\ \text { mar/07 } & 6 & 13 & 19 & 2.58 & 0 & 1 & 20 & 30 & 65 & 0 & 5 \\ \text { abr/07 } & 5 & 13 & 18 & 3.56 & 0 & 1 & 19 & 26.31 & 68.42 & 0 & 5.2 \\ \text { may/07 } & 4 & 15 & 19 & 6.37 * & 0 & 1 & 20 & 20 & 75 & 0 & 5 \\ \text { jun/07 } & 8 & 10 & 18 & 0.22 & 0 & 2 & 20 & 40 & 50 & 0 & 10 \\ \text { jul/07 } & 9 & 7 & 16 & 0.25 & 0 & 0 & 16 & 56.25 & 43.75 & 0 & 0 \\ \text { ago/07 } & 5 & 11 & 16 & 2.25 & 0 & 4 & 20 & 25 & 55 & 0 & 20 \\ \text { Total } & 72 & 136 & 208 & 19.69 * & 3 & 19 & 230 & 31.30 & 59.13 & 1.30 & 8.26 \\ \text { * } & & & & & & & & & & \end{array}$

\section{CUADRO 2}

Frecuencia absoluta y relativa de machos $(M)$, hembras $(F)$, hermafroditas $(H)$ y de sexo indeterminado $(I)$ del ostión Crassostrea rhizophorae en el Punto II-Isla del Tanque, septiembre 2006-agosto de 2007

TABLE 2

Absolute and relative frequency of the male $(M)$, female $(H)$, hermaphrodite $(E)$ and indeterminate sex $(I)$ of the Crassostrea rhizophorae oyster in Point II-Tanque Island, during from September 2006-August 2007

\begin{tabular}{lcccccccccccc}
\multicolumn{1}{c}{ Mes/Año } & $\mathrm{M}$ & $\mathrm{H}$ & $\mathrm{M}+\mathrm{H}$ & $\chi^{2}$ & $\mathrm{E}$ & $\mathrm{I}$ & Total & $\% \mathrm{M}$ & $\% \mathrm{H}$ & $\% \mathrm{E}$ & $\% \mathrm{I}$ \\
sep/06 & 6 & 11 & 17 & 1.47 & 0 & 2 & 19 & 31.57 & 57.9 & 0 & 10.5 \\
oct/06 & 6 & 14 & 20 & 3.2 & 0 & 0 & 20 & 30 & 70 & 0 & 0 \\
nov/06 & 6 & 13 & 19 & 2.58 & 1 & 0 & 20 & 30 & 65 & 5 & 0 \\
dec/06 & 7 & 12 & 19 & 1.31 & 0 & 0 & 19 & 36.84 & 63.15 & 0 & 0 \\
ene/07 & 10 & 10 & 20 & 0 & 0 & 0 & 20 & 50 & 50 & 0 & 0 \\
feb/07 & 6 & 13 & 19 & 2.58 & 1 & 0 & 20 & 30 & 65 & 5 & 0 \\
mar/07 & 6 & 14 & 20 & 3.2 & 0 & 0 & 20 & 30 & 70 & 0 & 0 \\
abr/07 & 6 & 14 & 20 & 3.2 & 0 & 0 & 20 & 30 & 70 & 0 & 0 \\
may/07 & 3 & 16 & 19 & $8.89^{*}$ & 0 & 3 & 22 & 13.63 & 72.72 & 0 & 13.6 \\
jun/07 & 7 & 13 & 20 & 1.8 & 0 & 0 & 20 & 35 & 65 & 0 & 0 \\
jul/07 & 6 & 14 & 20 & 3.2 & 0 & 0 & 20 & 30 & 70 & 0 & 0 \\
ago/07 & 3 & 13 & 16 & $6.25^{*}$ & 0 & 1 & 17 & 17.64 & 76.47 & 0 & 5.8 \\
Total & 72 & 157 & 229 & $31.55^{*}$ & 2 & 6 & 237 & 30.38 & 66.24 & 0.84 & 2.5
\end{tabular}

*valor significativo a nivel de $5 \%$ de probabilidad. 

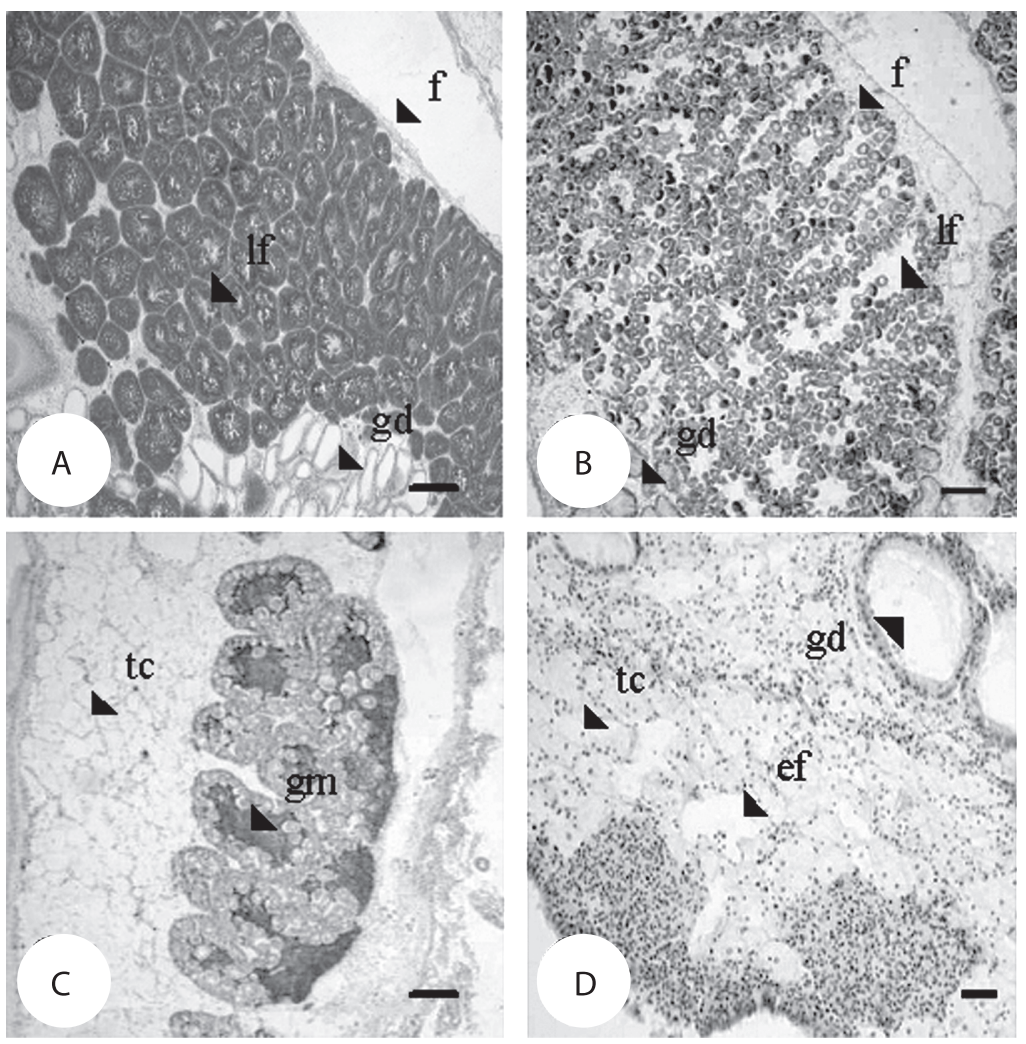

Fig. 2. Gónadas del ostión Crassostrea rhizophorae del estero del Río Maraú, Bahia, Brasil. A) macho, B) hembra, C) hermafrodita, D) sexo indeterminado; ef esbozo del folículo, f folículos, lf luz del folículo, gf gametos femeninos, gd glándula digestiva, gm gametos masculinos, tc tejido conectivo. Barra: A, B, C=100 $\mu \mathrm{m}$ y $\mathrm{D}=25 \mu \mathrm{m}$.

Fig. 2. Oyster gonad Crassostrea rhizophorae of the Maraú Estuary River, Bahia, Brasil. A) male, B) female, C) hermaphrodite, D) indeterminate sex; ef follicle outline, f follicle, lf follicle lumen, gf female gametes, gd digestive glandule, gm male gametes, tc connective tissue. Scale: A, B, C=100 $\mu \mathrm{m}$ and $\mathrm{D}=25 \mu \mathrm{m}$.

ocurrió de septiembre 2006 a marzo 2007 y de mayo a agosto de 2007. La recuperación de las gónadas ocurrió en noviembre de 2006, febrero a marzo y julio de 2007 (Fig. 3a y 3b).

Los diferentes estadios de madurez gonádica componen las figuras 4 y 5 .

Rendimiento de carne: Los valores de rendimiento de carne $(\mathrm{R})$ variaron de 8.0 a $17.7 \%$ en ejemplares con altura entre 30.4 y $94.6 \mathrm{~mm}$, con promedio de $46.9 \mathrm{~mm} \pm 8.8$ en el Punto I y $46.1 \mathrm{~mm} \pm 8.9$ en el Punto II (Fig. 6). Los promedios porcentuales más altos de $\mathrm{R}$ en el Punto I ocurrieron en octubre de 2006 y en enero, febrero, abril y agosto de 2007, con mayor registro en abril y un promedio de $15.9 \pm 3.3$ y en el Punto II, en octubre de 2006, febrero a agosto de 2007, con mayor registro en mayo de 2007 y un promedio de 17.8 44.2 .

\section{DISCUSIÓN}

Los ostiones del género Crassostrea son hermafroditas secuenciales sin dimorfismo sexual, pueden presentar uno u otro sexo y esta alternancia ocurre de acuerdo con la edad y 

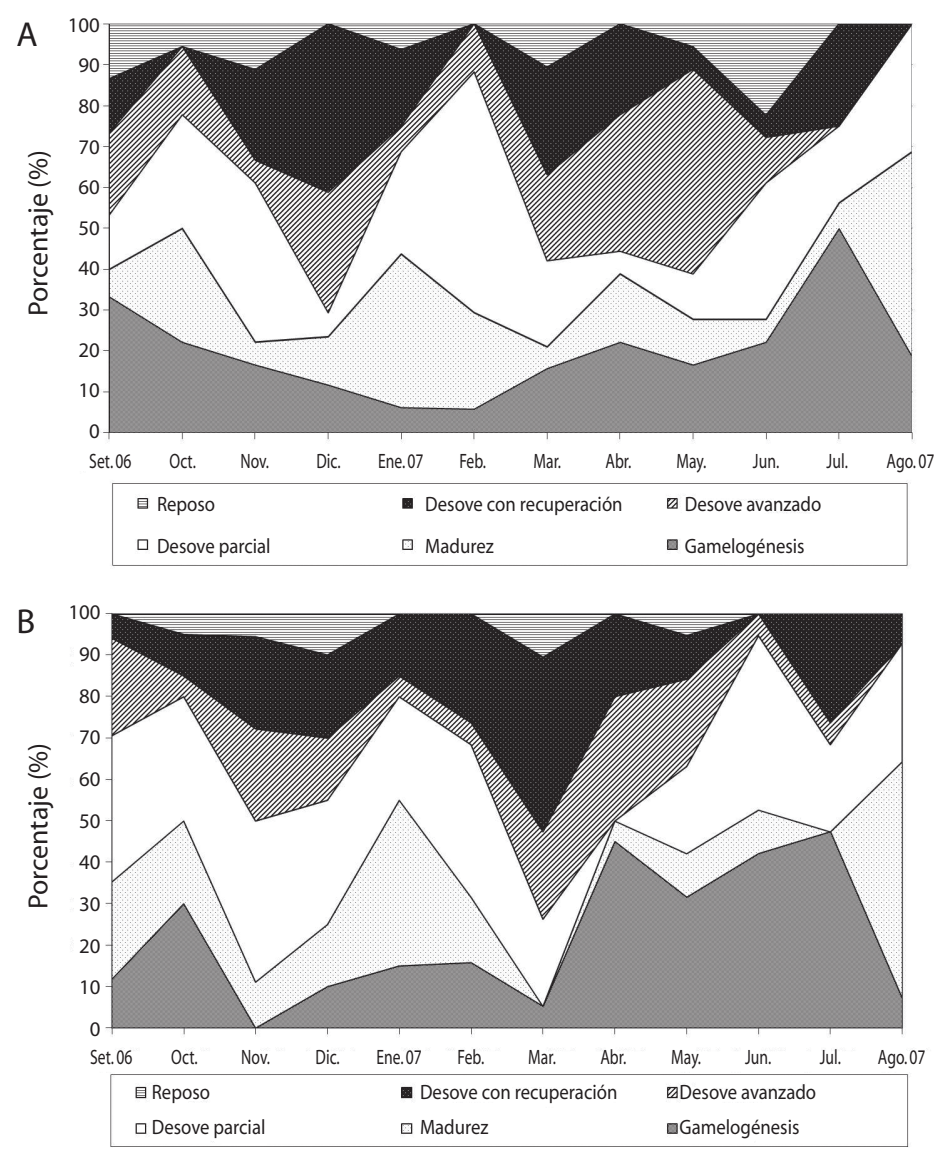

Fig. 3. Ciclo gonádico del ostión Crassostrea rhizophorae en el (a) Punto I-Maraú (n=208) e (b) en el Punto II-Isla del Tanque (n=229) agosto 2006-septiembre 2007.

Fig. 3. Gonadic cycle of the oyster Crassostrea rhizophorae at (a) Point I-Maraú (n=208) and (b) Point II-Tanque Island ( $n=229)$ from August 2006-September 2007.

las condiciones ambientales vigentes (Galtsoff 1964, Wakamatsu 1973, Andrews 1979).

La razón sexual de los ostiones $C$. rhizophorae en este estudio estuvo dominado por las hembras, similar a lo informado en estudios anteriores efectuados en otras localidades de la costa brasileña (Nascimento 1978, Christo 2006). Por lo general hubo una tendencia de mayor número de hembras en la entrada del estero (Punto II), sin embargo, la proporción de hembras no demostró diferencia significativa entre los puntos. Le Dantec (1968) sugirió que la proporción de hembras de Crassostrea angulata es mayor en regiones más próximas al océano, que en regiones menos salinas. Los ostiones en regiones más salinas, que muestra el menor crecimiento (Newkirk \& Field 1990), parecen disponer de más energía para fenómenos de la reproducción, lo que sugiere una mayor inversión en la acumulación de reservas, principalmente para la oogénesis.

La diferencia significativa encontrada entre el número de machos y hembras correspondió a períodos con gran número de animales en 

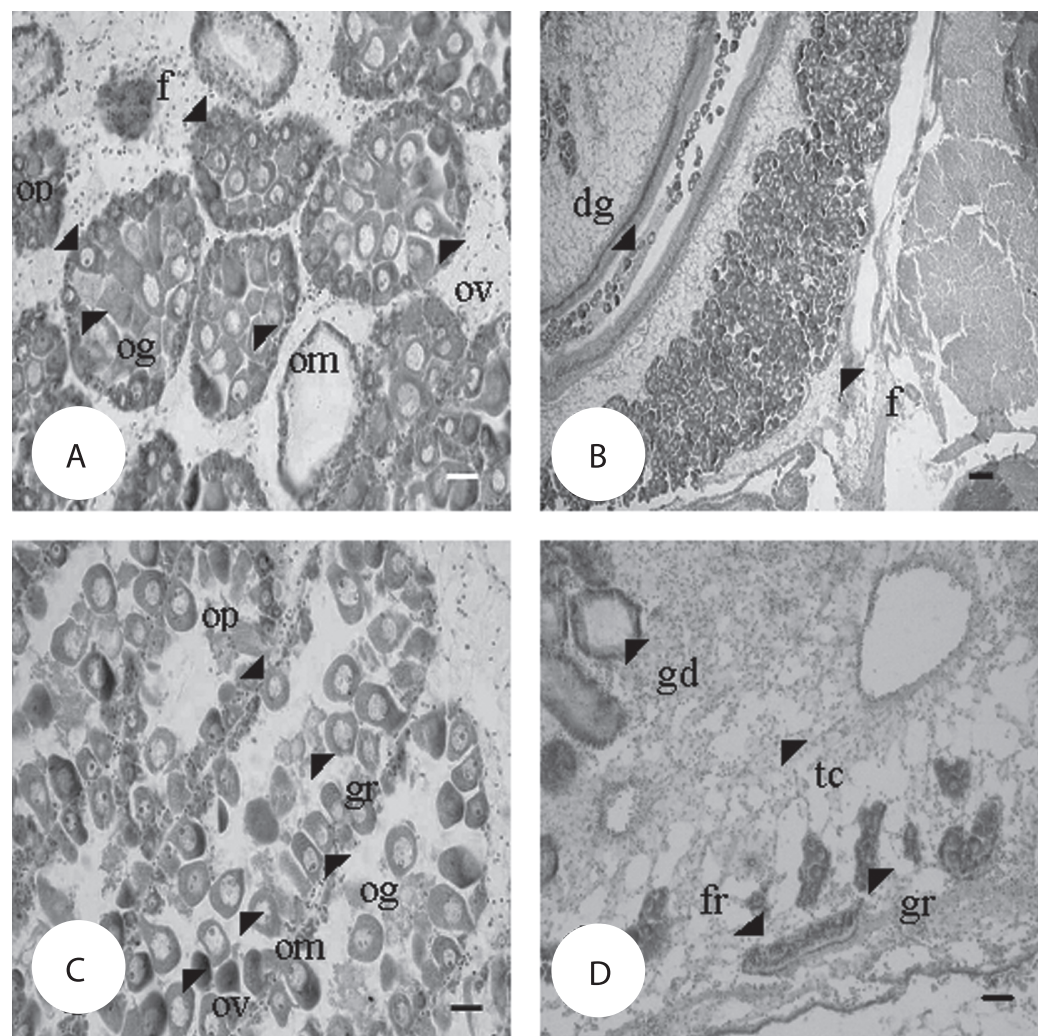

Fig. 4. Crassostrea rhizophorae hembra del estero del Río Maraú, Bahia, Brasil. Fases del desarrollo gonádico. A) madurez, B) desove parcial, C) desove con proliferación, D) reposo; dg ducto genital, f folículo, fr folículo en regresión, gr gametos residuales, og oogonia, om oocito maduro, op oocito previtelogénico, ov oocito vitelogénico, tc tejido conectivo. Barra: $25 \mu \mathrm{m} ; \mathrm{C}=100 \mu \mathrm{m}$.

Fig. 4. Female of Crassostrea rhizophorae of the Maraú River estuary, Bahia, Brasil. Development of the gonadial phases. A) fully ripe, B) post-spawning, C) spawning with proliferation, D) gamete residues; dg genital duct, $\mathbf{f}$ follicle, fr regressive follicle, gr remaining gametes, og oogonia, om rip oocyte, op oocyte pre-reserve yolk, ov oocyte reserve yolk, tc connective tissue. Scale: $25 \mu \mathrm{m}$; C: $100 \mu \mathrm{m}$.

estado de madurez y desove de los gametos, este hecho, pudo estar relacionado a la estrategia reproductiva del género Crassostrea. Las condiciones ambientales determinan la madurez de las gónadas y el proceso de desove, así como el tiempo de recuperación de las gónadas que exige un gasto energético mayor en las hembras que en los machos, ya que a oogénesis depende de una considerable reserva orgánica (Galtsoff 1964, Lunetta 1969, Andrews 1979). Con la recuperación más rápida de las gónadas, a cada estímulo ambiental favorable al desove, un individuo macho puede liberar gametos con mayor frecuencia y cantidad que un organismo hembra. Si la mayoría de los individuos invierten energía para la oogénesis, aumenta las oportunidades de fecundación de los gametos y consecuentemente de éxito reproductivo de la especie.

Una pequeña frecuencia de gónadas hermafroditas se encontró en una proporción similar a la reportada para especies tropicales de Crassostrea. Angell (1986) resaltó que entre 0.3 a $1.6 \%$ de ejemplares de C. rhizophorae 

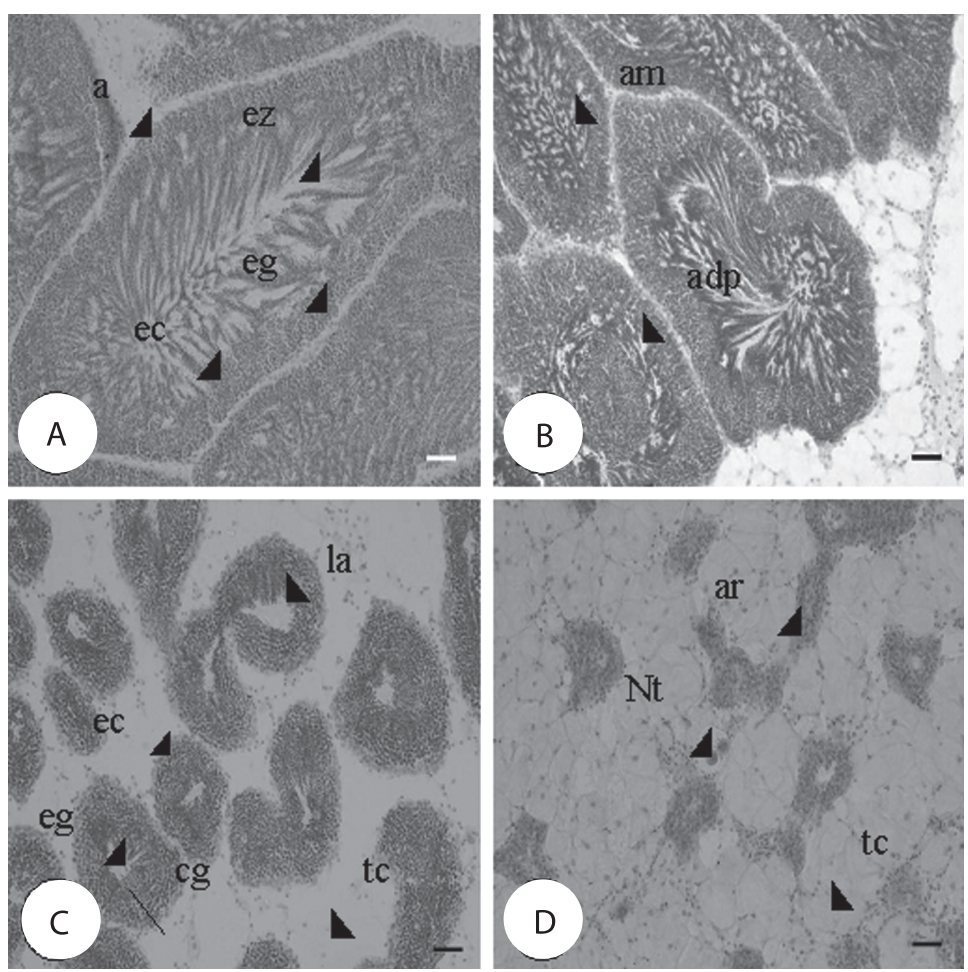

Fig. 5. Crassostrea rhizophorae macho en el estero del Río Maraú, Bahia, Brasil. Fases del desarrollo gonádico. A) madurez, B) desove parcial, C) desove con proliferación, D) reposo; a ácino gonádico, am ácino maduro, ar ácino em regresión, cg capa germinativa, eg espermatogônias, es espermatócitos, et espermátides, ez espermatozoides, la luz del ácini, Nt parásito Nematopsis sp., tc tejido conectivo. Barra: $25 \mu \mathrm{m}$.

Fig. 5. Male of Crassostrea rhizophorae in Maraú Estuary River, Bahia, Brasil. Development of the gonadial phases. A) fully ripe, B) post-spawning, C) spawning with proliferation, D) gamete residues; a gonadial acino, am fully rip acino, ar reabsorption acino, cg germinate layer, eg spermatogonias, es spermatocyte, et spermatids, ez spermatozoa, la acino lumen, Nt parasite Nematopsis sp., tc connective tissue. Scale: $25 \mu \mathrm{m}$.

presentan gónadas hermafroditas. Los meses en que se observó hermafroditismo fueron noviembre y febrero, que también son los períodos de mayor precipitación pluviométrica. La influencia de las variables ambientales, entre ellas la lluvia y, consecuentemente, la salinidad, en el proceso de determinación sexual han sido descritas en trabajos anteriores (Galtsoff 1964, Lunetta \& Grotta 1982). Dinamani (1974) sugirió que individuos de C. glomerata menores de un año predominan los machos y arriba de esta edad las hembras. El hermafroditismo pudo estar relacionado a la edad de los organismos y los estímulos ambientales que determinaron el momento del cambio sexual.

Con relación a los individuos de sexo indeterminado, fue registrada una baja incidencia, pero mayor de lo que encontrado por Nascimento (1978) en C. rhizophorae, que correspondió al 0,6\% de la población. Según la autora, después del proceso avanzado del desove, la gónada puede entrar nuevamente en estado de madurez o puede alcanzar la fase de indeterminación sexual. La mayor ocurrencia de individuos de sexo indeterminado en el sector interno del estero (Punto I) puede estar relacionada principalmente con las condiciones 


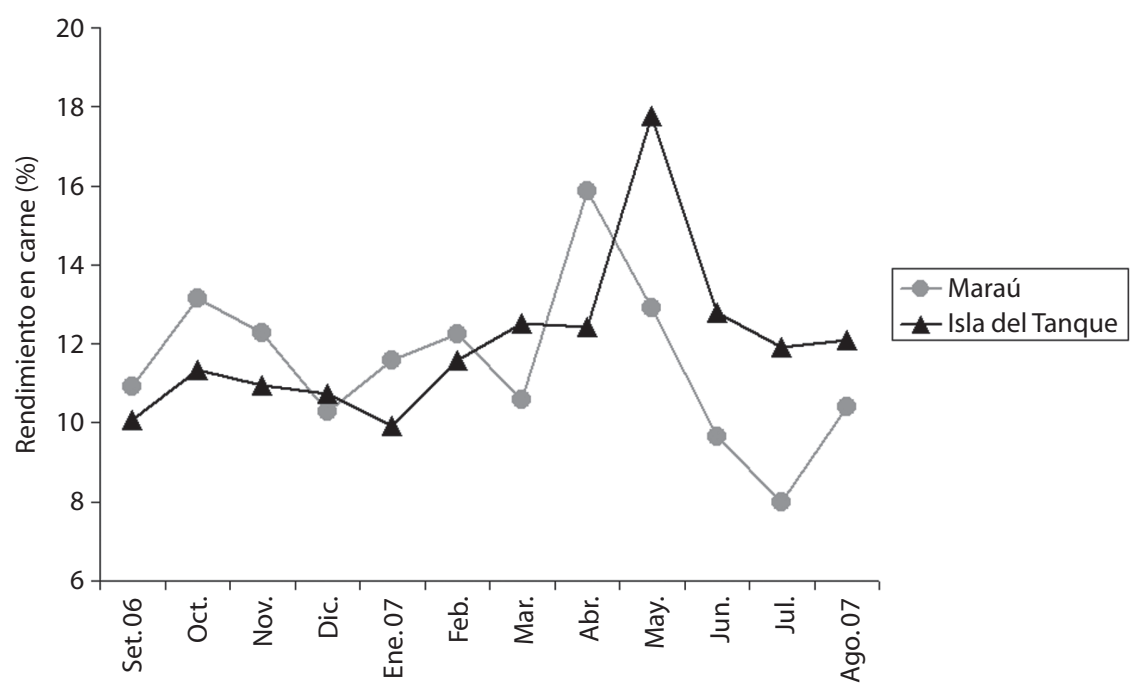

Fig. 6. Variación media mensual del rendimiento de carne (R) de las ostras Crassostrea rhizophorae colectadas en Maraú (Punto I, n=340) e Isla del Tanque (Ponto II, n=329), septiembre 2006-agosto 2007.

Fig. 6. Mean monthly variation of meat index (R) of the Crassostrea rhizophorae oysters collected in Maraú (Point I, n=340) and Tanque Island (Point II, n=329), from September 2006-August 2007.

más bajas de salinidad de ese sitio. Según una revisión realizada por Grotta \& Lunetta (1982), grandes variaciones en la salinidad influyen sobre el metabolismo y con el proceso de diferenciación sexual en los moluscos.

Nascimento (1978) sugiere que la liberación de gametos no es concomitante para todos los ostiones y que el desove es un proceso lento e individuos con desove parcial ocurren durante todo el año, principalmente después de los eventos de desove. En el estero del Río Maraú se observaron individuos en los diferentes estadios del ciclo gonádico durante todo el año.

Después, de los meses que presentaron la mayor pluviosidad en la región (octubre y febrero) se siguió una disminución en la frecuencia de ostiones en desove y un aumento en la frecuencia de individuos con proliferación de gametos (noviembre y marzo). La disminución de la salinidad ocurrida como consecuencia de las fuertes lluvias pudo haber estimulado el desove. Oocitos de ostiones que viven en condiciones de salinidad muy baja
$(<10)$, frecuentemente tienen rompimiento y remoción de las células de la gónada (Galtsoff 1964). En este caso, el momento del desove indicó ser estimulado por la caída de salinidad. Zamora et al. (2003), encontraron que el ostión americano $C$. virginica en México, tiene una alta correlación entre la salinidad y los eventos reproductivos de gametogénesis y desove. De acuerdo con otros autores (Jmeliova \& Sanz 1969, Angell 1986), el efecto de la salinidad puede también reflejarse en cambios de la calidad nutricional y abundancia de alimento.

La pequeña variación anual de temperatura parece haber sido el principal factor determinante en la continuidad del ciclo reproductivo en el estero del Río Maraú. El proceso de reproducción continuo durante el año fue anteriormente observado en ostiones (Vélez 1977, Nascimento 1978, Zamora et al. 2003, Cárdenas et al. 2007). Bacon (1971) indicó que pequeñas variaciones anuales en la temperatura, asociadas con condiciones físicas favora- 
bles, son los principales factores que propician la reproducción durante la mayor parte del año.

El R (rendimiento) fue mayor en abril y mayo (Punto I y II, respectivamente). Galvão et al. (2000) observaron que el $\mathrm{R}$ es mayor en los individuos en gametogénesis y maduros, debido a que en estas fases ocurren la acumulación de reservas y el pleno desarrollo de los folículos. Esto aunado a los dos estadios de desarrollo gonádico, los meses de mayor $\mathrm{R}$, presentaron el $40 \%$ de los individuos con gran acumulo de reservas, en ambos sitios. Los mayores registros de $\mathrm{R}$, encontrados en octubre de 2006, enero y febrero de 2007 en el Punto I y en febrero y mayo hasta julio de 2007 en el Punto II, fueron también observados en los análisis histológicos de las gónadas.

El momento de mayor desove indicó ser estimulado por la caída de salinidad. La pequeña variación de temperatura propicia la reproducción durante la mayor parte del año, lo que posibilita una constante captación de semillas y su cultivo en el estero del Río Maraú.

\section{AGRADECIMIENTOS}

Este estudio fue realizado bajo el patrocinio del proyecto "Bioecologia, Enfermidades e Implantação de Cultivos de Ostras e Sururus na Baía de Camamu, BA" Chamada Pública MCT/FINEP/SEAP-Aqüicultura-Ação Transversal $n^{\circ} 12 / 2005$ (Brasil). Agradecemos el apoyo de la Fundação de Amparo à Pesquisa do Estado da Bahia (FAPESB) por la concesión de la beca. A la Organização em Pró-Defesa e Estudos dos Manguezais da Bahia (ORDEM), y en especial a Elias Leal Veloso, por el apoyo logístico para la realización del trabajo. A Saul E. Méndez Sánchez por la revisión del manuscrito en español.

\section{RESUMEN}

El ostión de manglar es un importante recurso pesquero que se distribuye por toda la costa brasileña y una de las especies nativas con mayor potencial para maricultura. Este estudio tuvo como objetivo conocer las características reproductivas del ostión de manglar Crassostrea rhizophorae en el estero del Río Maraú, Bahía de Camamu, Bahia, Brasil. Las muestras fueran recolectadas mensualmente, entre agosto de 2006 y septiembre de 2007, en dos sitios. Las metodologías utilizadas fueron el análisis histológico de las gónadas y el cálculo de rendimiento de carne. La temperatura del agua durante el período de estudio varió de $23.5^{\circ} \mathrm{C}$ a $30^{\circ} \mathrm{C}$ y la salinidad osciló entre 15 y 35 ups. La altura de los ostiones examinados varió de 27 a 102mm $(n=437)$. Hubo predominancia de hembras en las poblaciones de ambos sitios. Los análisis de estadios gonádicos demostraron que el proceso de reproducción de $C$. rhizophorae en el estero del Río Maraú es continuo durante el año, sin período de reposo sexual. El rendimiento de carne $(\mathrm{R})$ fue de $8.0 \%$ a $17.7 \%(\mathrm{n}=669)$. Los resultados de este estudio proveen informaciones para la captación de semilla e implantación de la ostricultura en la Bahía de Camamu.

Palabras clave: Crassostrea, ostión de manglar, reproducción, rendimiento, ostricultura, Brasil.

\section{REFERENCIAS}

Andrews, J.D. 1979. Pelecypoda: Ostreidae, p. 293-341. In A.C. Giese \& J.S. Pearse (eds.). Reproduction of Marine Invertebrates. Vol. V: Molluscs: Pelecypods and Lesser Classes. Academic, Nueva York, EEUU.

Angell, C.L. 1986. The biology and culture of tropical oyster. ICLARM, Manila, Filipinas.

Bacon, P.R. 1971. Studies on the biology and cultivation of the mangrove oyster in Trinidad with notes on the other shellfish resource. Trop. Sci. 4: 265-278.

Cardenas, E.R.B., D.A. Aranda, M.L. Sevilla \& P.F.R. Espinosa. 2007. Variations in the reproductive cycle of the oyster Crassostrea virginica (Gmelin, 1791), Pueblo Viejo lagoon, Veracruz, Mexico. Transit. Waters Bull. 2: 37-46.

Christo, S.W. 2006. Biologia reprodutiva e ecologia de ostras do gênero Crassostrea Sacco, 1897 na Baía de Guaratuba (Paraná-Brasil): um subsídio ao cultivo. Tesis Doctorado, Universidade Federal do Paraná, Curitiba, Paraná, Brasil.

Clédon, M., J.L. Brichtova, J.L. Gutiérrez \& P.E. Penchaszadeh. 2004. Reproductive cycle of the stout razor clam, Tagelus plebeius (Lightfoot, 1786), in the mar Chiquita coastal lagoon, Argentina. J. Shellfish Res. 2: 443-446.

Dinamani, P. 1974. Reproductive cycles and gonadial changes in the New Zealand rock oyster Crassostrea conglomerata. J. Mar. Freshwater Res. 8: 34-65. 
EPAGRI. Empresa de Pesquisa Agropecuária e Extensão Rural do Estado de Santa Catarina. 2007. Síntese informativa da produção de moluscos (mexilhões, ostras e vieiras) no Estado de Santa Catarina em 2006. (Consultado 20 octubre 2007, www.epagri. ret-sc.br).

Galtsoff, P.S. 1964. The American oyster Crassostrea virginica (Gmelin). U.S. Department of the Interior Fish and Wildlife Serv., USA

Galvão, M.S.M., O.M. Pereira, I.C. Machado \& M.B. Henriques. 2000. Aspectos reprodutivos da ostra Crassostrea brasiliana de manguezais do estuário de Cananéia, SP $\left(25^{\circ} \mathrm{S}-48^{\circ} \mathrm{W}\right)$. B. Inst. Pesca 2: 147-162.

Jmeliova, N.N. \& J. Sanz. 1969. Respiración y algunas particularidades de la alimentación del ostión Crassostrea rhizophorae (Guilding, 1828). Ser. Oceanol. 3: $1-20$.

Le Dantec, J. 1968. Reproduction em Crassostrea angulata Lmk dans le Basin D' Arcachon. Quelques comparaisons avec les huitres de la Gironde. Rev. Trav. Inst. Pech. Marit. 32: 300-362.

Lunetta, J.E. 1969. Fisiologia da reprodução dos mexilhões (Mytilus perna- Mollusca-Lamellibranchia). Bol. Fac. Filos. Cienc. Universidade São Paulo 26: 33-111.

Lunetta, J.E. \& M. Grotta. 1982. Influência de fatores exógenos e endógenos sobre a reprodução de moluscos marinhos. Bol. Fisiol. Animal Universidade São Paulo 6: 191-204.

Nascimento, I.A. 1978. Reprodução da ostra do mangue, Crassostrea rhizophorae (Guilding, 1828): um subsídio ao cultivo. Tesis de doctorado, Universidade de São Paulo, San Pablo, Brasil.

Nascimento, I.A. 1983. Cultivo de ostras no Brasil: Problemas e perspectivas. Cienc. Cult. 7: 871-876.

Nascimento, I.A. \& J.E. Lunetta. 1978. Ciclo sexual da ostra de mangue e sua importância para o cultivo. Bol. Fisiol. Animal Universidade de São Paulo 2: 63-93.

Nascimento, I.A. \& S.A. Pereira. 1980a. Change in the condition index from mangrove oysters (Crassostrea rhizophorae) from Todos os Santos Bay, Salvador, Brasil. Aquaculture 20: 9-15.

Nascimento, I.A. \& S.A. Pereira. 1980b. Efeito do caranguejo Pinnotheres ostreum em ostras Crassostrea rhizophorae. Bol. Inst. Oceanogr. Universidade São Paulo 2: 261-265.

Nascimento, I.A. \& S.A. Pereira. 2004. Cultivo da ostra de mangue Crassostrea rhizophorae (Guilding, 1828), p. 267-288. In C.R. Poli, A.T. Poli, E. Andreatta \& E. Beltrame (eds.). Aqüicultura: Experiências Brasileiras. UFSC-Multitarefa, Florianópolis, Brasil.

Nascimento, I.A., E.M. Silva, M.I.S. Ramos \& A.E. Santos. 1980. Desenvolvimento da gônada primária em ostras do mangue Crassostrea rhizophorae: idade e tamanho mínimo de maturação sexual. Cienc. Cult. 6: $736-742$

Newkirk, G.F. \& B.A. Field. 1990. Oyster culture in the Caribbean. Halifax: International Development Research Center.

Pereira, O.M., M.B Henriques \& I.C. Machado. 2003. Estimativa da curva de crescimento da ostra Crassostrea brasiliana em bosques de mangue e proposta para sua extração ordenada no estuário de Cananéia, SP, Brasil. B. Inst. Pesca 1: 19-28.

Prieto, A.S., M.S. Flores \& C. Lodeiros. 1999. Madurez sexual e índice de condición en una población del mejillón de fondo Modiolus squamosus (Mollusca, Bivalvia) en Tocuchare, Golfo de Cariaco, Venezuela. Ecotropica 12: 83-90.

Rebelo, M.F., M.C.R. Amaral \& W.C. Pfeiffer. 2005. Oyster condition index in Crassostrea rhizophorae (Guilding, 1828) from a heavy-metal polluted coastal lagoon. Braz. J. Biol. 2: 345-351.

Rios, E.C. 1994. Seashells of Brasil. Editora de la Fundación Universidad Rio Grande, Rio Grande, Brasil.

Ruiz, J.B., J. Benítez \& T. Okuda. 1969. Variación estacional de la composición química del ostión, Crassostrea rhizophorae (Guilding) en la Laguna Grande y la Bahía Mochima. Bol. Inst. Oriente Venezuela 1-2: 46-52.

S.E.I. - Superintendência de Estudos Econômicos e Sociais da Bahia. 2000. Censo 2000. Base Cartográfica Digital, CD-ROM.

Shaw, B.L. \& H.I. Battle. 1957. The gross and microscopic anatomy of the digestive tract of the oyster Crassostrea virginica (Gmelin). Can. J. Zool. 1: 325-347.

Souza-Lima, W., C.L.C. Manso, E.J.E. Andrade \& J.L. Gri1lo. 2003. Bacias Sedimentares Brasileiras, Bacia de Camamu. Fundação Paleontológica Phoenix, Editora Interciência, Rio de Janeiro, Brasil. 
Vélez, A. 1976. Crecimiento, edad y madurez sexual del ostión Crassostrea rhizophorae de Bahía de Mochima. Bol. Inst. Oriente Venezuela 1: 65-72.

Vélez, A. 1977. Ciclo anual de reproducción del ostión Crassostrea rhizophorae (Guilding) de Bahía de Mochima. Bol. Inst. Oriente Venezuela 1-2: 87-98.
Wakamatsu, T. 1973. A ostra de Cananéia e o seu cultivo. SUDELPA, Instituto Oceanográfico, Universidade de São Paulo. San Pablo, Brasil.

Zamora, A., M.L.S. Hernández \& D.A. Aranda. 2003. Ciclo gonádico del ostión americano Crassostrea virginica (Lamellibranchia: Ostreidae) en Mecoacán, Tabasco, México. Rev. Biol. Trop. 51: 109-117. 